\title{
Towards prediction of ordered phases in rechargeable battery chemistry via group-subgroup transformation
}

\author{
Yunbing Ran ${ }^{1}$, Zheyi Zou ${ }^{2}$, Bo Liu ${ }^{3}$, Da Wang ${ }^{1}$, Bowei Pu ${ }^{1}$, Penghui Mi ${ }^{4}$, Wei Shi ${ }^{1}$, Yajie Li ${ }^{1}$, Bing He $\mathbb{D}^{4}$, Ziheng Lu ${ }^{5}$, Xia Lu ${ }^{6}$, \\ Baihai $\mathrm{Li}\left(\mathbb{D}^{7}\right.$ and Siqi Shi (iD)
}

The electrochemical thermodynamic and kinetic characteristics of rechargeable batteries are critically influenced by the ordering of mobile ions in electrodes or solid electrolytes. However, because of the experimental difficulty of capturing the lighter migration ion coupled with the theoretical limitation of searching for ordered phases in a constrained cell, predicting stable ordered phases involving cell transformations or at extremely dilute concentrations remains challenging. Here, a group-subgroup transformation method based on lattice transformation and Wyckoff-position splitting is employed to predict the ordered ground states. We reproduce the previously reported $\mathrm{Li}_{0.75} \mathrm{CoO}_{2}, \mathrm{Li}_{0.8333} \mathrm{CoO}_{2}$, and $\mathrm{Li}_{0.8571} \mathrm{CoO}_{2}$ phases and report a new $\mathrm{Li}_{0.875} \mathrm{CoO}_{2}$ ground state. Taking the advantage of Wyckoff-position splitting in reducing the number of configurations, we identify the stablest $\mathrm{Li}_{0.0625} \mathrm{C}_{6}$ dilute phase in Li-ion intercalated graphite. We also resolve the $\mathrm{Li} / \mathrm{La} /$ vacancy ordering in $\mathrm{Li}_{3 x} \mathrm{La}_{2 / 3-x} \mathrm{TiO}_{3}(0<x<0.167)$, which explains the observed Li-ion diffusion anisotropy. These findings provide important insight towards understanding the rechargeable battery chemistry.

npj Computational Materials (2021)7:184; https://doi.org/10.1038/s41524-021-00653-y

\section{INTRODUCTION}

The ever-growing demands for electrical energy storage have led to the higher performance requirements for rechargeable batteries $^{1-5}$. Tremendous efforts have been devoted to the study of (i) high voltage or capacity cathodes (e.g., $\mathrm{Li}\left[\mathrm{Ni}_{1-y-z} \mathrm{Mn}_{y} \mathrm{Co}_{z}\right] \mathrm{O}_{2}, \mathrm{NMC}$ compounds) $)^{6,7}$, (ii) solid electrolytes (e.g., garnet, perovskite, and NASICON family) ${ }^{8-15}$, and (iii) post-lithium battery chemistries (e.g., $\mathrm{Na}, \mathrm{K}$, and $\mathrm{Mg}$ batteries) ${ }^{16-22}$. A key commonality of the above electrolytes and electrodes is that their properties (e.g., ionic conductivity for electrolytes, phase stability, and voltage for electrodes) are closely linked to the concentrations of mobile ions and the corresponding ordered ground states during either the preparation or ion-intercalation process. Unfortunately, it is difficult to determine the ordered ground states in these systems because of the low sensitivity of current spectroscopic techniques to the light elements (e.g., $\mathrm{H}$ and $\mathrm{Li})^{13}$. For example, even neutron scattering cannot detect the precise occupation of $\mathrm{Li}^{+}$in $\mathrm{Li}^{-}$ containing compounds, only giving a "disordered" distribution of these ions in an averaged manner. Thus, it is difficult to directly obtain the precise arrangements of mobile ions at the atomic scale. For example, the exact ordered ground states of the lithium graphite intercalation compounds (LGICs), a commercially successful graphite anode, at their dilute limit (viz., $\mathrm{Li}_{x} \mathrm{C}_{6}$ with $0<x<0.5$ ) and the accurate arrangements of $\mathrm{Li} / \mathrm{La} /$ vacancy in $\mathrm{Li}_{3 x} \mathrm{La}_{2 / 3-x} \mathrm{TiO}_{3}$ (LLTO, $0<x<0.167$ ), a solid electrolyte, are still under debate ${ }^{23,24}$. Therefore, it is critical to resolving the ordered ground states of the electrodes or solid electrolytes during the operation with the help of foresighted calculations.

In this context, the theoretical lattice-gas model (LGM) ${ }^{25,26}$ is widely used to determine the $\mathrm{Li}^{+}$occupation ordering in guesthost intercalation electrochemical systems, where the host is an ordered-sites containing network and each site can be occupied by a guest or vacancy. Various configurations associated with rechargeable battery chemistries can be obtained by enumerating possible mobile ion/vacancy arrangements on the given supercell, which is produced by replication of the unit cell with a specified integer number ${ }^{27-29}$. To overcome the enormous configurational space challenge or avoid the large-scale first-principles calculations, several so-called "parameter-constructing" methods, including sampling-based Metropolis Monte Carlo and fitting effectivecluster-interaction-based cluster expansion, etc., have been developed ${ }^{30-32}$. The most representative method is the cluster expansion (CE). It sets up a mathematical framework where the energy is expanded as a series of cluster basis functions that can be multiplied by effective cluster interactions (ECls). However, prediction errors will inevitably occur when a large lattice mismatch among configurations and small training sets of configurations are encountered ${ }^{33}$.

The problem of the ordered arrangements of alkali-ion/vacancy in alkali-ion batteries mentioned above can be treated as a group-subgroup transformation (viz., reducing symmetry from the parent structure) since all possible arrangements can be classified into one of the subgroups. Different from supercells which are determined ad hoc, structures obtained by group -subgroup transformation have their lattices which are determined by the transformation matrix. Thus, all possible supercells can be included. Moreover, unlike randomizing or enumerating the arrangements of alkali-ion/vacancy, group-subgroup transformation assigns alkali-ion/vacancy into distinct subsets of Wyckoff positions in each subgroup to avoid enumeration ${ }^{34-36}$. Therefore, ordered phases within diverse supercells and dilute concentrations can be formulated rigorously.

\footnotetext{
${ }^{1}$ School of Materials Science and Engineering, Shanghai University, Shanghai 200444, China. ${ }^{2}$ School of Materials Science and Engineering, Xiangtan University, Xiangtan, Hunan 411105, China. ${ }^{3}$ College of Mathematics and Physics, Jinggangshan University, Ji'an, Jiangxi 343009, China. ${ }^{4}$ School of Computer Engineering and Science, Shanghai University, Shanghai 200444, China. ${ }^{5}$ Department of Materials Science \& Metallurgy, University of Cambridge, Cambridge CB30FS, UK. ${ }^{6}$ School of Materials, Sun Yat-sen University, Guangzhou Higher Education Mega Center, Guangzhou 510006, China. ${ }^{7}$ School of Materials and Energy, University of Electronic Science and Technology of China, Chengdu 610054, China. ${ }^{8}$ Materials Genome Institute, Shanghai University, Shanghai 200444, China. ${ }^{\bowtie}$ email: sqshi@shu.edu.cn
} 
Previously, this method has been employed to find the relationship among the high symmetric original structures in existing ordered phases ${ }^{34-36}$. In this context, we describe a prediction framework based on such a group-subgroup transformation for generating possible ordered phases of electrolyte/ electrodes with variable concentrations of mobile ions during the typical preparation/ion-intercalation process. High-precision firstprinciples formation energies are further employed to determine the ordered ground states. Potential variation, e.g., stacking of the host lattice, is also included for comparison. By searching through a comprehensive range of supercells, we identify the commonly accepted ordered ground states of $\mathrm{Li}_{x} \mathrm{CoO}_{2}$ and propose $\mathrm{Li}_{0.875} \mathrm{CoO}_{2}$ as a new ordered ground state at $x>0.75$. Moreover, utilizing the Wyckoff position splitting approach to reduce the number of configurations, the extremely dilute $\mathrm{Li}_{0.0625} \mathrm{C}_{6}$ phase can be identified. By extending our group-subgroup transformation method to uncover the joint ordering of $\mathrm{Li} /$ vacancies with immobile $\mathrm{La}$ in the solid electrolyte $\mathrm{Li}_{3 x} \mathrm{La}_{2 / 3-x} \mathrm{TiO}_{3}$, we reveal that the Li-ion diffusion anisotropy is caused by the blocking effect of La ions.

\section{RESULTS}

\section{Constructing convex hull of $\mathrm{Li}_{x} \mathrm{CoO}_{2}$ within transformed lattices}

Because the ordered ground states in $\mathrm{Li}_{x} \mathrm{CoO}_{2}$ determine the $0 \mathrm{~K}$ equilibrium voltage, understanding the ordering of Li/vacancy in Li-de-intercalated phases is important for tailoring this material to the specific electrochemical application. In the exploration of $\mathrm{Li}_{x} \mathrm{CoO}_{2}$ ordered ground states during the charging/discharging process, the first and most important step is to determine the possible configurations. To cover as many configurations as possible, the most common method is to search within several ad hoc supercells. However, the results may conflict with each other when different supercells are used. For example, Van der Ven et al. concluded that $\mathrm{Li}_{0.8333} \mathrm{CoO}_{2}$ is one of the ordered ground states predicted by the cluster-expansion method ${ }^{37}$, whereas Wolverton et al. suggested that $\mathrm{Li}_{0.8571} \mathrm{CoO}_{2}$ is the ordered ground state ${ }^{38}$. This discrepancy might stem from the use of different sets of supercells. Herein, using the group-subgroup transformation method, the ordering of $\mathrm{Li} /$ vacancy in $\mathrm{Li}_{x} \mathrm{CoO}_{2}$ is studied by searching for configurations with different Li concentrations via enumerating different sizes of supercells and obtaining ordered phases from transformed lattices, thus avoiding possibly missing stable configurations. In principle, the group-subgroup transformation method should enable us to obtain diverse supercells based on the symmetry reduction and lattice transformation for $\mathrm{Li}_{x} \mathrm{CoO}_{2}$, where different $\mathrm{Li}$ concentrations are examined.

It is worth noting that the rearrangement of the $\mathrm{LiCoO}_{2}$ host structure occurs by altering the stacking sequences of oxygen during charge and discharge. The only difference between the original and rearranged structures is how the $\mathrm{O}-\mathrm{Co}-\mathrm{O}$ slabs of $\mathrm{LiCoO}_{2}$ relate to each other across the Li planes. Because ordered phases are determined within a specific host structure, we must consider these different host structures as an additional variable in addition to the Li concentrations. Experimentally, three hosts have been confirmed. The first is $\mathrm{O}^{39}$, which has $\mathrm{ABC}$ oxygen stacking while the second is $\mathrm{O} 1$ and has an $A B A B$ oxygen stacking ${ }^{40}$. The stability of the $\mathrm{O} 1$ host is demonstrated to be restricted to zero $\mathrm{Li}$ concentration. The third is referred to as $H 1-3^{41,42}$, which features the characteristics of both $\mathrm{O} 3$ and $\mathrm{O} 1$. $\mathrm{Li}$ is assumed to prefer the octahedral sites of $\mathrm{O} 3$ to those of $\mathrm{O} 1$. Thus, the maximum $\mathrm{Li}$ concentration that can be obtained in this host with $x=0.5$ in $\mathrm{Li}_{x} \mathrm{CoO}_{2}$. Supplementary Table 1 presents the detailed structural information of these hosts. The group-subgroup transformation method is applied to each of these hosts. Motivated by the experimental observations, we only consider trigonal and monoclinic candidate subgroups ${ }^{35}$. Finally, formation energies of 377 nonidentical configurations are used to determine the ordered ground states.

As illustrated in Fig. 1, the $\mathrm{O} 1-\mathrm{CoO}_{2}$ host is more stable with lower formation energy ( $60 \mathrm{meV}$ per f.u.) than that of the $\mathrm{O} 3$ host at zero $\mathrm{Li}$ concentration. This result is in good agreement with the previously reported values of $40^{37}$ and $50 \mathrm{meV}^{38}$. This is why in electrochemical experiments, it is difficult to obtain single $\mathrm{O} 3$ $-\mathrm{CoO}_{2}$ with $0 \mathrm{Li}$ in the structure. Several ground states at various $\mathrm{Li}$ concentrations are identified, as indicated by the convex hull formed by seven stable phases at $x=0.1667,0.3333,0.5,0.6667$, $0.75,0.3333$, and 0.875 . Detailed structural information is provided in Supplementary Table 2. Our computation reveals that the $\mathrm{O} 3$ host is stable above $x=0.3333$. Below such a value, the $H 1-3$ host is stable, and an ordered ground state is observed at $x=0.1667$.

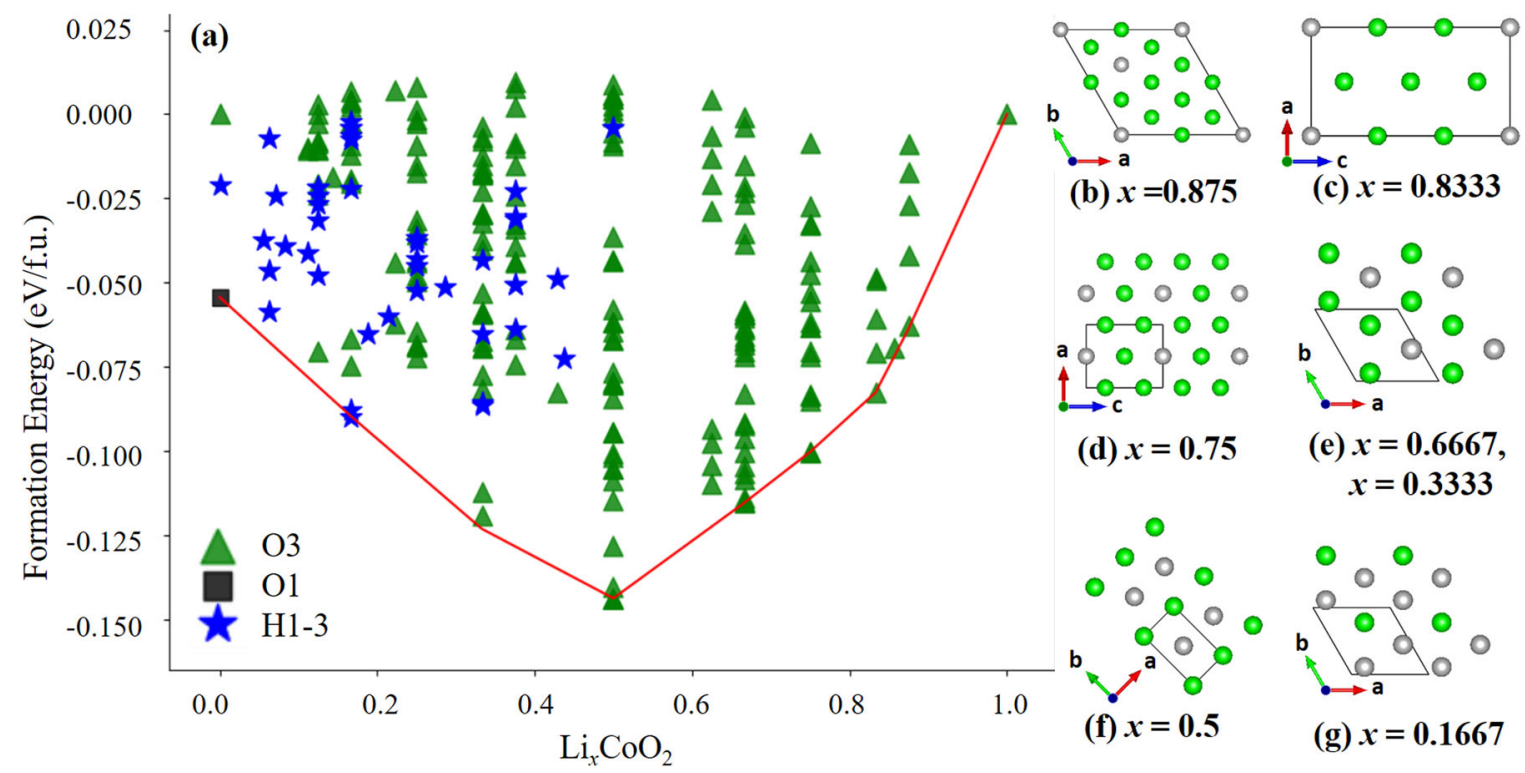

Fig. 1 Convex hull of $\mathrm{Li}_{x} \mathrm{CoO}_{2}(\mathbf{0}<\boldsymbol{x}<\mathbf{1})$. a Formation energies (per formula unit, $\Delta E_{\mathrm{f}}$ ) of $\mathrm{Li}_{x} \mathrm{CoO}_{2}$. Ordered ground states at $x=\mathbf{b} 0.875$, c 0.8333 , d 0.75 , e $0.6667,0.3333, \mathbf{f} 0.5, \mathbf{g} 0.1667$. Energies greater than $10 \mathrm{meV}$ are discarded. The green and gray balls represent Li atoms and vacancies, respectively. 


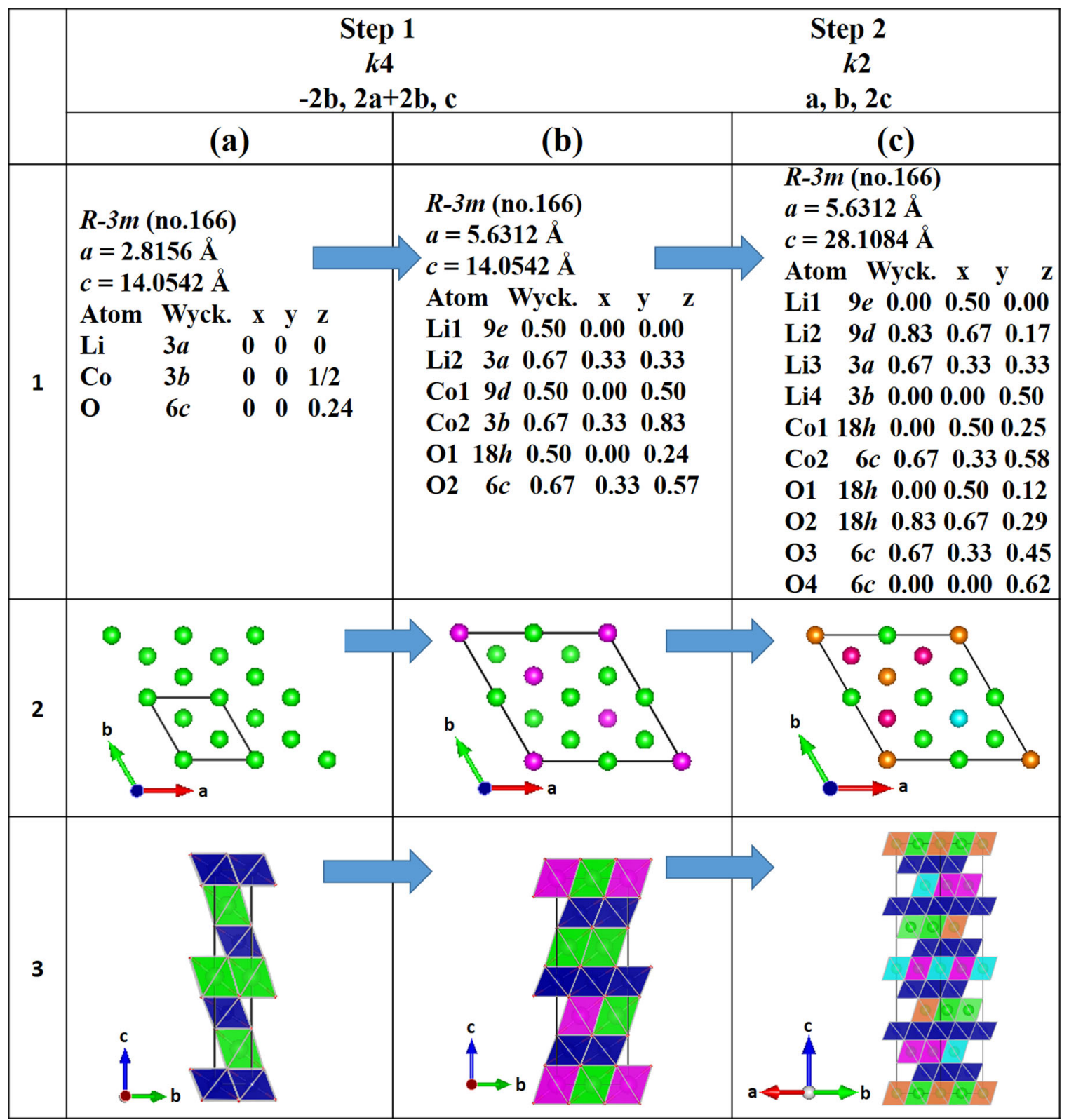

Fig. 2 Phase transitions from $\mathrm{LiCoO}_{2}$ to $\mathrm{Li}_{0.875} \mathrm{CoO}_{2}$. a $\mathrm{LiCoO}_{2}$ original structure. $\mathbf{b}$ Isomorphic enlargement with an index of 4 in ab plane. c Isomorphic enlargement with an index of 2 along $\mathrm{C}$ axis. Li1, Li2, Li3, and Li4 are colored in green, pink, yellow, and cyan, respectively.

In the following section, we describe each stable ordered phase obtained by the group-subgroup transformation method in detail.

To ensure the validity of the group-subgroup transformation method, we present the ordering pattern of Li/vacancies in $\mathrm{Li}_{0.5} \mathrm{CoO}_{2}$, which has in-plane $2 \times 1$ ordering with the Li-ions arranged in rows and separated by rows of vacancies (Supplementary Table 2). This arrangement was initially proposed by Reimers and Dahn based on in situ X-ray powder-diffraction measurements ${ }^{43}$ and was later confirmed by Shao-Horn based on electron diffraction experiments ${ }^{35}$. Van der Ven et al. ${ }^{37}$ also obtained this arrangement using the cluster-expansion method and confirmed this ordering. In our work, this arrangement is obtained by subgroup $P 2 / m$ with lattice transformation of $2 / 3 \mathbf{a}+1 /$ $3 \mathbf{b}-2 / 3 \mathbf{c}, \mathbf{b}, \mathbf{c}$. In this subgroup, the Li sites ( $3 a)$ split into $1 a$ and $1 e$ subsets, and one of them is extracted. The ordered phases at $x=0.1667,0.3333$, and 0.6667 are also verified. They have the same $\sqrt{3} \times \sqrt{3}$ ordering in the $\sqrt{3} \times \sqrt{3}$ trigonal lattice with space group $P 3_{1} 12$ (Supplementary Fig. 1), which is consistent with the theoretical prediction and experimental observation at $-170^{\circ} \mathrm{C}^{35,37,38}$. Numerous arrangements within different supercells are compared, and they all indicate that $\sqrt{3} \times \sqrt{3}$ ordering is the most stable one (Supplementary Table 3 ). This provides a reasonable explanation for why these orderings are successfully confirmed in different works.

It is worth noting that at $x>0.75$, there is no consensus on whether $x=0.8333$ or $x=0.8571$ is the ground state ${ }^{37,38}$. This inconsistency results from the differences in the sizes of ad hoc supercells. Here, ordered phases in different supercells with sizes up to eight times of the primitive cell are conducted for the systematic investigation. The ordered phases at $x=0.8333$ and 0.8571 mentioned above are completely included in $\sqrt{3} \times 3$ and $\sqrt{7} \times \sqrt{7}$ supercells within $k 6$ and $k 7$ subgroups, respectively (Supplementary Table 4). Figure 1 shows that the ordered ground states are $\mathrm{Li}_{0.8333} \mathrm{CoO}_{2}$ and $\mathrm{Li}_{0.875} \mathrm{CoO}_{2}$. The energy of $\mathrm{Li}_{0.8571} \mathrm{CoO}_{2}$ slightly leaves the tie line between $x=0.75$ and $x=0.875$ with a value of $3 \mathrm{meV}$. 


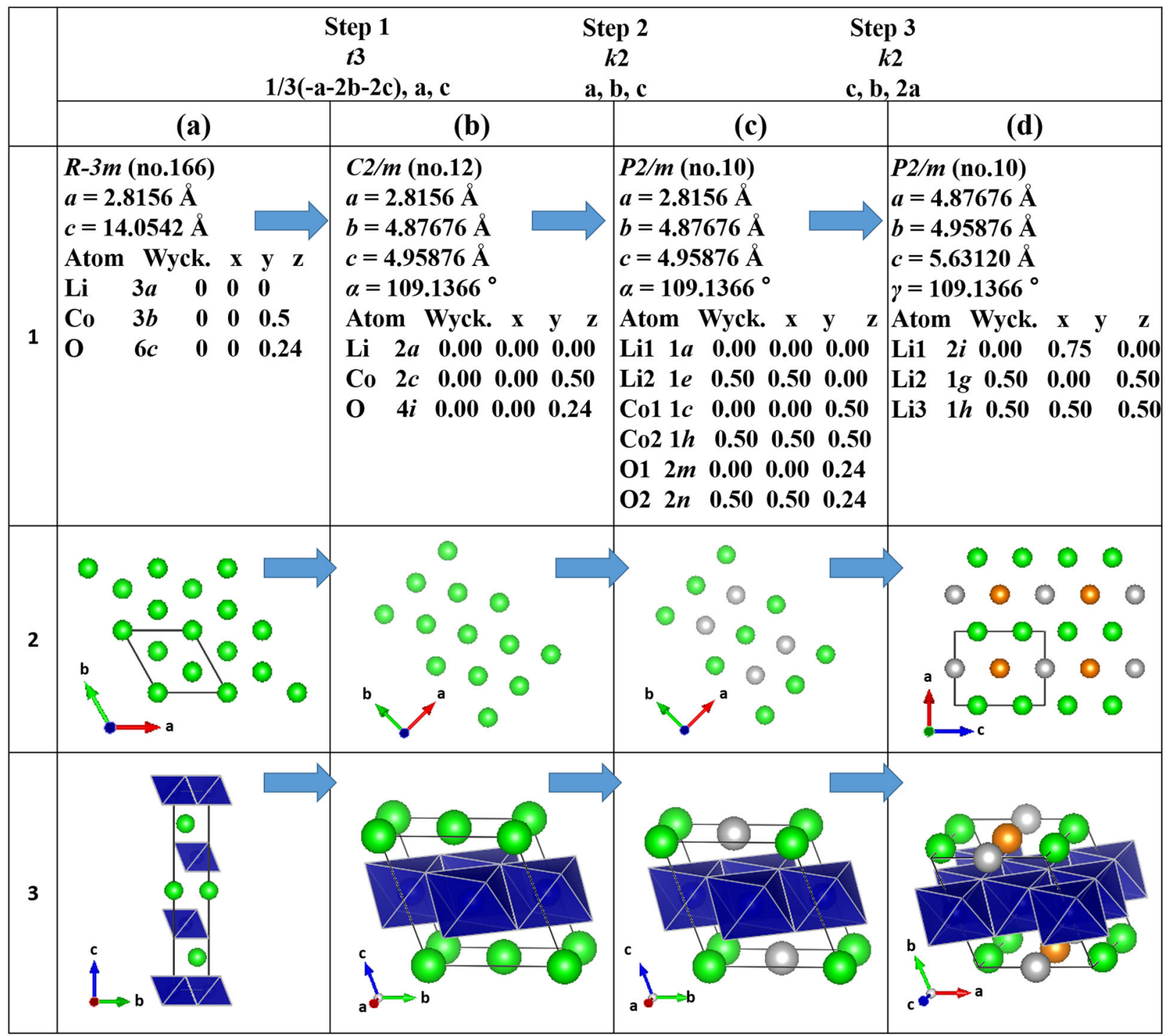

Fig. 3 Phase transitions from $\mathrm{LiCoO}_{2}$ to $\mathrm{Li}_{0.75} \mathrm{CoO}_{2}$. a $\mathrm{LiCoO}_{2}(R-3 m)$. b Obtaining $\mathrm{C} 2 / \mathrm{m}$ by loss of -3 symmetry operation. c Obtaining $P 2 / m$ by loss of centering. d Isomorphic enlargement of $P 2 / m$ with an index of 2 . All the structures are converted to the reduced cells. The blue octahedron represents the $\mathrm{CoO}_{6}$ polyhedron.

Figure 2 shows the transformation from $\mathrm{LiCoO}_{2}$ to the $\mathrm{Li}_{0.875} \mathrm{CoO}_{2}$ within a $2 \times 2 \times 2$ supercell. This structure is obtained by isomorphic enlargement of the $\mathrm{O}_{3}-\mathrm{LiCoO}_{2}$ with a $k$-index of 8 . First, the cell is transformed using the lattice transformation $-2 \mathbf{b}$, $2 \mathbf{a}+2 \mathbf{b}, \mathbf{c}$ in the ab plane (Fig. $2 b$ ). Then, the $2 c$ transformation is applied, as shown in Fig. 2c. In this subgroup structure, the Li can occupy the $9 e(0,0.5,0), 9 d(0.83333,0.6667,0.1667), 3 a(0.6667$, $0.3333,0.3333)$, and $3 b(0,0,0.5)$ positions, respectively. Comparison of the positions of lithium in both $\mathrm{LiCOO}_{2}$ and $\mathrm{Li}_{0.875} \mathrm{CoO}_{2}$ indicates that the phase transition occurs after the extraction of $\mathrm{Li}$ at $3 a(0.6667,0.3333,0.3333)$ or $3 b(0,0,0.5)$, which also confirms the suitability of this method for determining the ordered ground states with different Li concentrations in different supercells.

We also investigate the ordered ground state at $x=0.75$, where phases with $2 \times 2$ and $2 \times 4$ ordering have been reported previously ${ }^{34}$. By considering different supercells, both orderings are obtained by the group-subgroup transformation. Our calculation shows that the $2 \times 2$ ordering phase is less stable with an energy of $20 \mathrm{meV}$ per f.u. higher than that of the most stable one. Thus, the $2 \times 4$ ordering is the ground state.
Figure 3 shows the subgroup evolution of the supercell of $2 \times 4$ ordering. Starting from the $\mathrm{O}_{3}-\mathrm{LiCOO}_{2}$, we obtained $\mathrm{C} 2 / \mathrm{m}$ from space group $R \overline{3} m$ by a $t$-index of 3 due to the loss of the -3 symmetry operation. Subsequently, a loss of $C$-centering leads to the formation of the $P 2 / m$ structure with a $k$-index of 2 . Finally, isomorphic enlargement with an index of 2 is applied. The final lattice transformation can be $-1 / 3 \mathbf{a}-2 / 3 \mathbf{b}-2 / 3 \mathbf{c}, 2 \mathbf{a}, \mathbf{c}$, and the reduced cell is adapted. This transformation leads the Wyckoff position of $\mathrm{Li}$ splitting to change from $3 a(0,0,0)$ to $2 i(0,0.75,0)$, $1 g(0.5,0,0.5)$, and $1 h(0.5,0.5,0.5)$. Each of the positions can be occupied by a vacancy. In this structure, the arrangement of lithium and vacancies has been selected in agreement with the previously predicted ordering by Van der Ven et al. and Wolverton et al. $^{37,41}$.

\section{Identifying of the dilute ordered $\mathrm{Li}_{x} \mathrm{C}_{6}$ phases}

The intercalation process of lithium in the layered materials often results in the formation of "stages", such as LGIC. These stages describe the $2 \mathrm{D}$ stacking sequence of the lithium layers between the graphene layers, e.g., stage $n$ contains $n$ empty graphene layers between each Li-filled layer. Similarly, different stages have 


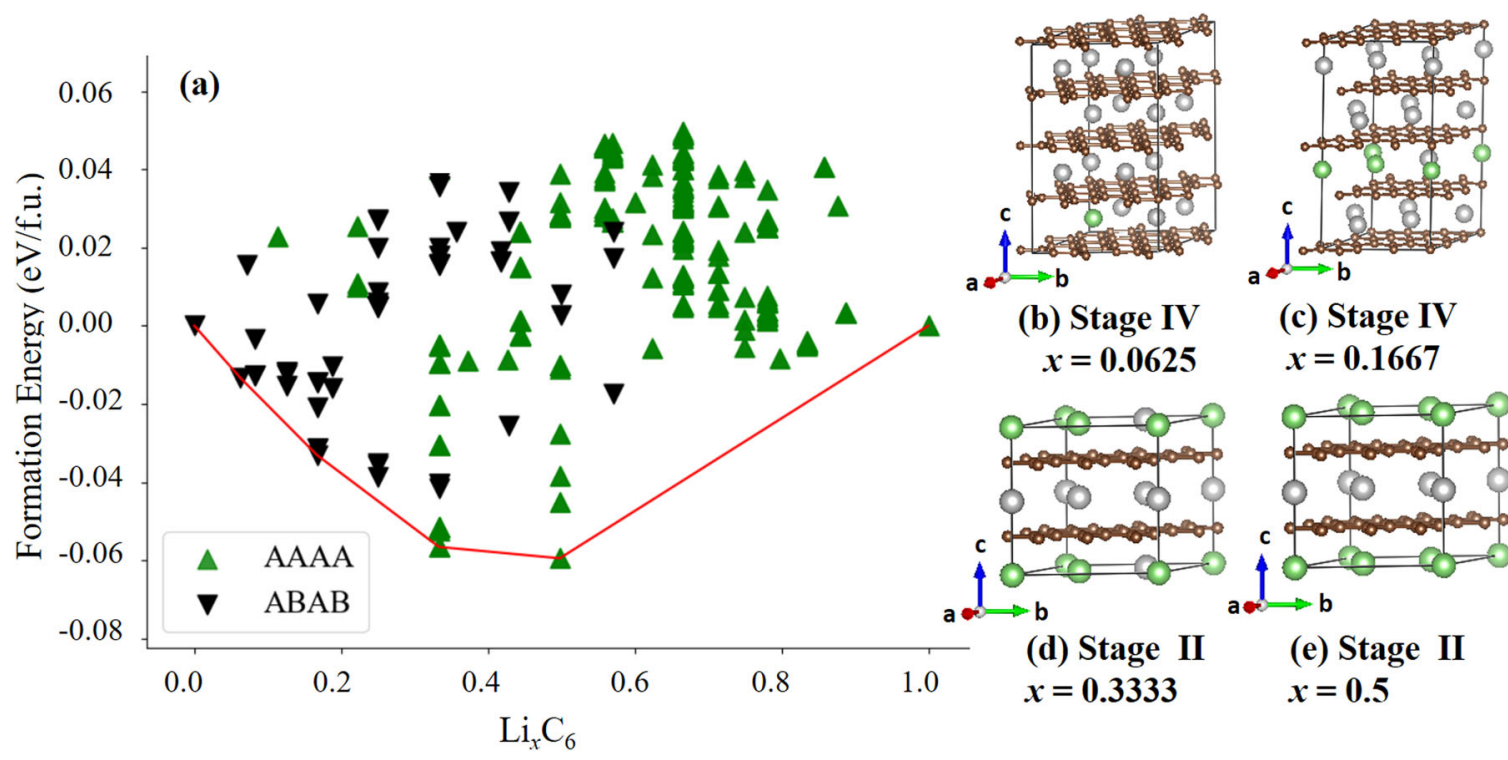

Fig. 4 Convex hull of $\mathrm{Li}_{x} \mathrm{C}_{6}(\mathbf{0}<\boldsymbol{x}<\mathbf{1})$. a Formation energies (per formula unit, $\left.\Delta E_{f}\right)$ of $\mathrm{Li}_{x} \mathrm{C}_{6}$ as a function of Li composition. Ordered ground states determined by the convex hull at $x=\mathbf{b} 0.0625$, c 0.1667 , d 0.3333, e 0.5. Energies larger than 50 meV are discarded.

\begin{tabular}{|c|c|c|c|c|c|}
\hline \multicolumn{2}{|c|}{$\begin{array}{l}\text { Step } 1 \\
k 2 \\
a, b, 2 \mathrm{c}\end{array}$} & \multicolumn{4}{|l|}{$\begin{array}{l}\text { Step } 2 \\
\quad 2 \\
a, b, c\end{array}$} \\
\hline 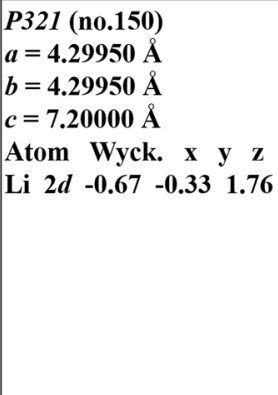 & 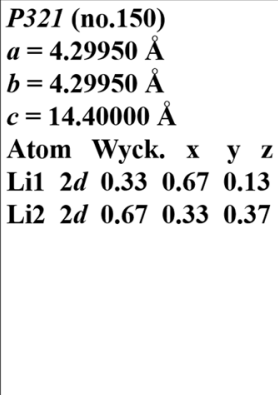 & 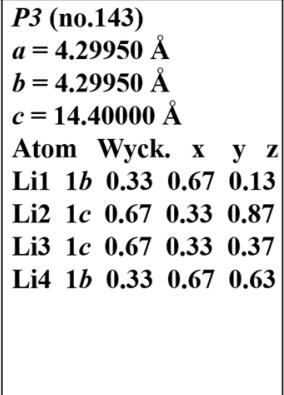 & $\begin{array}{c}\text { Step 3-1 } \\
k 3 \\
a-b, a+2 b, c\end{array}$ & 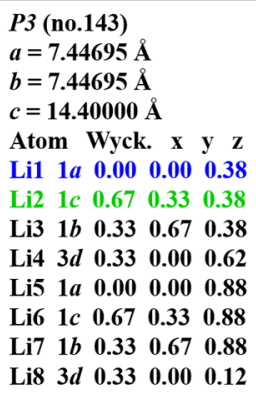 & $\underset{b \rightarrow \infty}{c}$ \\
\hline 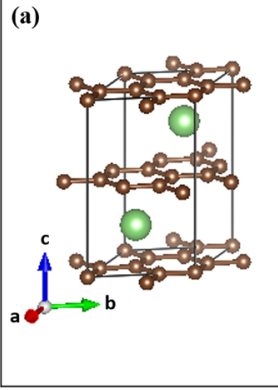 & (b) & (c) & $\begin{array}{c}\text { Step 3-2 } \\
k 4 \\
2 \mathrm{a}, 2 \mathrm{~b}, \mathrm{c}\end{array}$ & 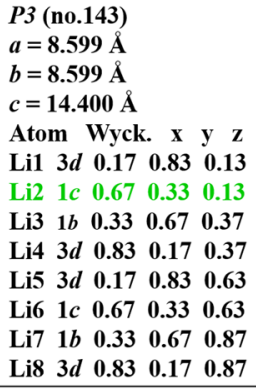 & $\stackrel{b}{a}$ \\
\hline
\end{tabular}

Fig. 5 Illustration of $\mathbf{L i}_{x} \mathbf{C}_{6}$ ordered phases with $\mathbf{0 . 0 6 2 5}, \mathbf{0 . 0 8 3 3 , 0 . 1 6 7} \mathbf{L i}$. a Parent structure of AB stacking $\mathrm{LiC}_{6}$ b Isomorphic enlargement by a, b, 2 c. c $P 3$ subgroup is obtained with $t$-index of 2 . $\mathbf{d ~} \mathrm{Li}_{0.0833} \mathrm{C}_{6}$ and $\mathrm{Li}_{0.1667} \mathrm{C}_{6}$ are obtained by $\mathbf{a}-\mathbf{b}, \mathbf{a}+2 \mathbf{b}, \mathbf{c}$. e $\mathrm{Li}_{0.0625} \mathrm{C}_{6}$ is obtained by $2 \mathbf{a}, 2 \mathbf{b}, \mathbf{c}$.

also been observed in $\mathrm{LiFePO}_{4}, \mathrm{KC}_{8}$, etc. using $\mathrm{XRD}$ and electrochemical techniques ${ }^{44,45}$. However, detailed structural information on stages with different Li concentrations from both experimental and theoretical studies is still scarce, leading to a non-unified description of such stages, especially for extremely dilute $\mathrm{Li}$ concentrations ${ }^{46-48}$. For example, the precise arrangement of atoms in each stage has rarely been reported either computationally or experimentally.

To evaluate the thermodynamic stability of the LGIC with various concentrations and stages, sequences with $A A$ and $A B$ stacking have been considered because of the relative gliding during charge and discharge processes ${ }^{49}$. For the former, graphene layers have a stacking order of $-\mathrm{A}-\mathrm{A}-\mathrm{A}-\mathrm{A}-$, while another stacking pattern is formed by shifting to a zigzag shape (-A-B-A-B-) (Supplementary Table 5). In the current work, we aim to use the group-subgroup transformation method and firstprinciples calculations to (1) search for the ordered ground states as a function of $\mathrm{Li}$ concentrations in the $\mathrm{Li}_{x} \mathrm{C}_{6}(0<x<1)$ system, (2) reproduce the experimental and theoretical studies on the stages of LGIC, and (3) obtain a clear picture of the ordered phases for dilute Li concentrations.

Firstly, we use group-subgroup transformation to search for possible configurations in the $\mathrm{Li}_{x} \mathrm{C}_{6}(0<x<1)$ system. Then, formation energies of 585 nonidentical configurations are obtained to determine the ordered ground states through first-principles calculations. Figure 4 shows that the transition 


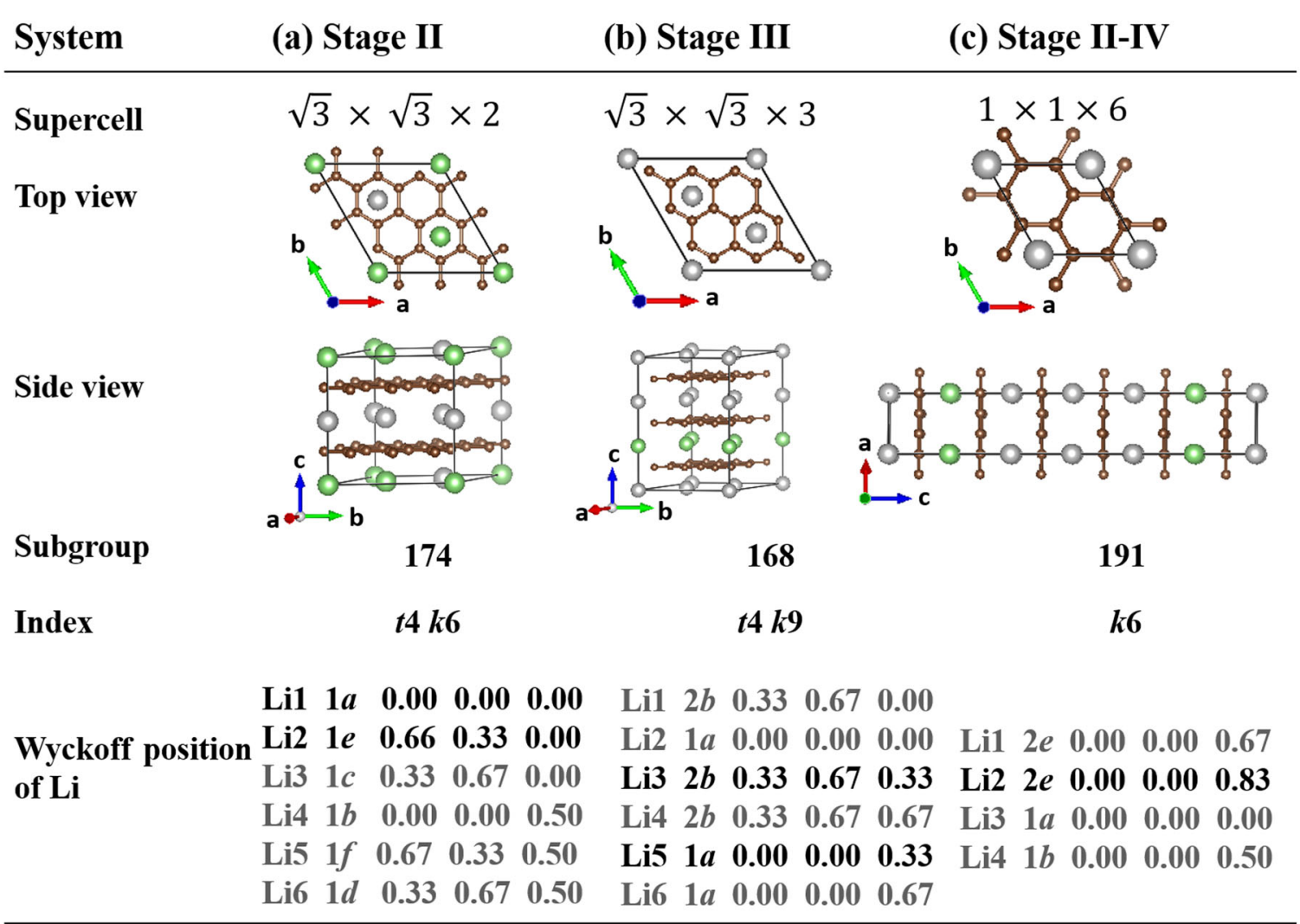

Fig. 6 Subgroup structures and Li/vacancy arrangements at $\mathrm{Li}_{0.3333} \mathrm{C}_{6}$. (a) stage II (b) stage III (c) stage II-IV. Subgroup structures and Li/ vacancy arrangements of stage $\mathrm{II}$, stage III, and stage II-IV at $\mathrm{Li}_{0.3333} \mathrm{C}_{6}$.

from $A B$ stacking to $A A$ stacking is facilitated by increasing the $\mathrm{Li}$ concentration, and the transition of stages occurs in the sequence of stage IV, stage II, and terminal $\mathrm{LiC}_{6}$ with a stage of I. The composition-induced stages occur at well-defined $x$ values of approximately $0.0625,0.1667,0.3333$, and 0.5 . Detailed information on these stages is provided in Supplementary Table 6.

Of these ordered ground states, the experimentally and computationally reported $\mathrm{Li}_{0.5} \mathrm{C}_{6}$ compound is well known for its stage II ordering ${ }^{50}$. Using the group-subgroup transformation method, we can compare the arrangements in several different supercells nearing the convex hull, such as $\sqrt{3} \times \sqrt{3} \times 2,2 \times 2 \times 2$, $1 \times 1 \times 4,1 \times 1 \times 6$. Results indicate that stage II is more energetically preferred than the other arrangements. It is generally accepted that phases with concentrations between 0.5 and 1 are coexisting phases (stage I and stage II), even at temperatures up to $\sim 200^{\circ} \mathrm{C}^{47,51}$.

In the dilute concentrations, because research on LGIC leads to a large number of configurations, brute force computation is not realistic. In previous research, the most stable dilute phase with $A B$ stacking was observed at $\mathrm{Li}_{0.0833} \mathrm{C}_{6}$, which has a stage of IV within a $\sqrt{3} \times \sqrt{3} \times 2$ supercell ${ }^{49}$. This phase is verified by our group-subgroup transformation when the $1 c$ site is occupied in the $P 3$ subgroup with the transformation matrix of $\mathbf{a}-\mathbf{b}, \mathbf{a}+2 \mathbf{b}, 2 \mathbf{c}$ (green color in Fig. 5d). With group-subgroup transformation, the total configurational space is in hundreds, avoiding the search for the ordered ground states in a space of more than $10^{4}$ configurations within the dilute region (Supplementary Fig. 2). Apart from this dilute phase at $\mathrm{Li}_{0.0833} \mathrm{C}_{6}$, we identify that a more dilute phase occurs at $\mathrm{Li}_{0.0625} \mathrm{C}_{6}$ with a stage of IV.

The ordered ground state of $\mathrm{Li}_{0.0625} \mathrm{C}_{6}$ has a subgroup of $P 3$ (no. 143 ) with transformation matrix $2 \mathbf{a}, 2 \mathbf{b}, 2 \mathbf{c}$. During this process, the site of $\mathrm{Li}$ in the parent structure splits from $2 d$ to $3 d, 1 c, 1 b, 3 d, 3 d$,
$1 c, 1 b$, and $3 d$ sites of the subgroup structure (Fig. 5e), and only the $1 c$ site is occupied by Li. It should be noted that the number of configurations obtained by group-subgroup transformation is greatly smaller than that obtained by enumeration, as illustrated in Supplementary Fig. 2. To understand the interaction between $\mathrm{Li}$ atoms in $\mathrm{Li}_{0.0625} \mathrm{C}_{6}$ and $\mathrm{Li}_{0.0833} \mathrm{C}_{6}$, we calculate the charge-density differences using the $2 \times 1 \times 1$ supercell (Supplementary Fig. 3). The charge-density difference profile shows that the blue isosurfaces only reside around a single $\mathrm{Li}$ atom in $\mathrm{Li}_{0.0625} \mathrm{C}_{6}$, which suggests that the $\mathrm{Li}$-atom interaction disappears in $\mathrm{Li}_{0.0625} \mathrm{C}_{6}$. These findings confirm that the $\mathrm{Li}_{0.0625} \mathrm{C}_{6}$ with extremely low $\mathrm{Li}$ concentration is the final extremely dilute concentration phase during the charging/discharging process.

We also note that there is non-consensus on the ground state at a Li concentration of $x=0.3333$, i.e., stage II, stage III, and stage II-IV have been proposed in various studies for this concentration $^{52,53}$. Using group-subgroup transformation, possible arrangements under different stages for $\mathrm{Li}_{0.3333} \mathrm{C}_{6}$ are determined within different supercells as shown in Fig. 6. Compared with the formation energies of stage III and stage II-IV, the value of stage II is lower by 30 and $40 \mathrm{meV}$ per $C_{6}$, respectively, indicating that the stage II phase of the $\mathrm{Li}_{0.3333} \mathrm{C}_{6}$ compound is more stable. This computational observation is consistent with the experimental results of Yazami et al. ${ }^{54}$. When comparing with the stage II structure of $\mathrm{Li}_{0.5} \mathrm{C}_{6}$, the Li layers of stage II are not fully occupied. Similar conflicts also appear at $\mathrm{Li}_{0.1667} \mathrm{C}_{6}$. This phase has the same subgroup as $\mathrm{Li}_{0.0833} \mathrm{C}_{6}$, with another $1 a$ site occupied (blue color in Fig. $5 \mathrm{~d}$ ). Moreover, when comparing with the stage II phase mentioned in previous work ${ }^{49}$, stage IV has lower formation energy of 20 meV per $\mathrm{C}_{6}$., which indicates that a higher stage is preferred in dilute $\mathrm{Li}$ concentrations. 

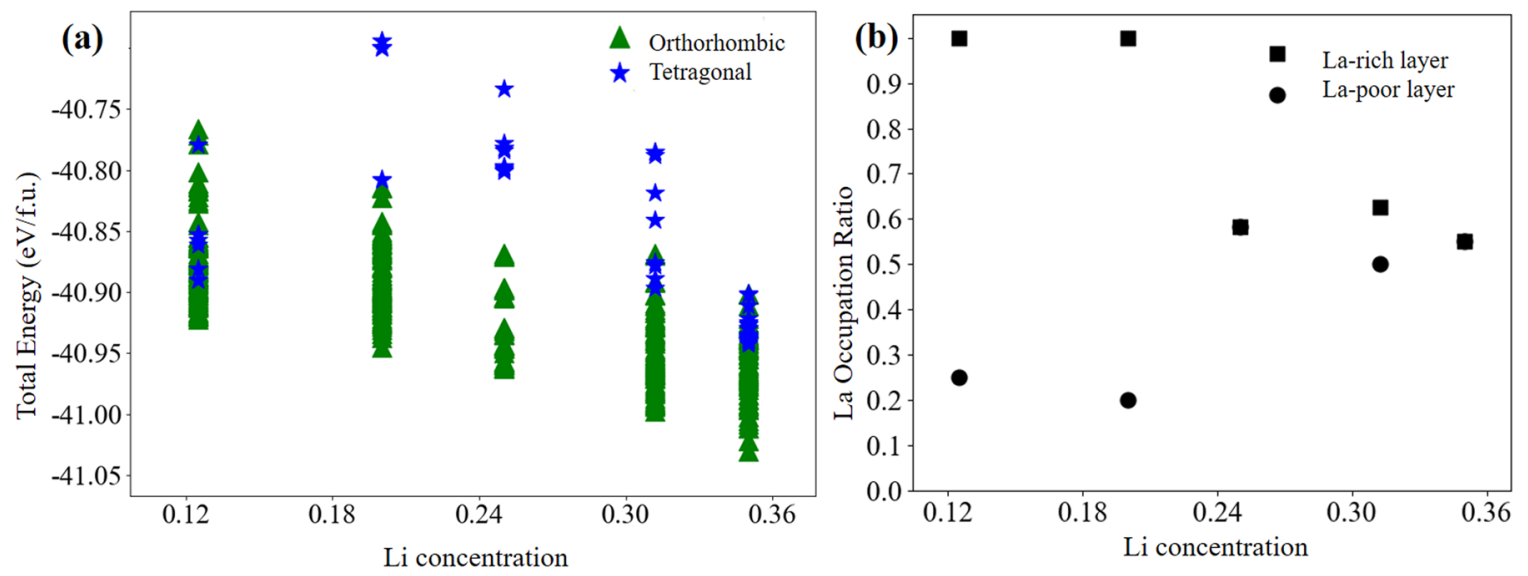

Fig. 7 Energy and ordering preference of $\mathrm{Li}_{3 x} \mathrm{La}_{2 / 3-x} \mathrm{TiO}_{3}$. a Comparison of the total energies at $3 x=0.125,0.2,0.25,0.3125$, and 0.35 within orthorhombic and tetragonal symmetries. b Averaged La ordering preference in ordered ground states.

\begin{tabular}{|c|c|c|c|}
\hline$x$ & $a$ & $b$ & c \\
\hline 0.125 & 0.234 & 0.830 & 9.268 \\
\hline 0.2 & 0.205 & 0.840 & 8.965 \\
\hline 0.25 & 9.102 & 0.840 & 0.215 \\
\hline 0.3125 & 9.277 & 0.293 & 0.205 \\
\hline 0.35 & 9.0527 & 0.293 & 0.293 \\
\hline
\end{tabular}

\section{Identifying $\mathrm{Li} / \mathrm{La} / \mathrm{vacancy}$ orderings in $\mathrm{Li}_{3 x} \mathrm{La}_{2 / 3-x} \mathrm{TiO}_{3}$}

Solid electrolytes have been widely studied because they are applicable in energy-dense solid-state batteries and other electrochemical devices. The elementary process of ionic transport is known to be strongly affected by the distribution of local ordering, i.e., the Li/vacancy ordering for Li-stuffed garnets ${ }^{13}$ and the more complicated coupled ordering of Li/vacancies with immobile $\mathrm{La}$ in $\mathrm{Li}_{3 x} \mathrm{La}_{2 / 3-x} \mathrm{TiO}_{3}$. In the latter case, the diffusion pathways are strongly affected by the La ordering ${ }^{55-57}$. In previous work, to elucidate the effect of La on Li diffusion, the initial Li/La/vacancy ordering has been specified ad hoc ${ }^{55,58}$. To address the La ordering rigorously, an effective guiding tool is needed. Inspired by the effectiveness in predicting ordered phases within transformed lattices, the group-subgroup method is naturally employed to determine the blocking effect of La on the Li diffusion pathway. Here, the starting structures are generated by simultaneously assigning $\mathrm{Li} / \mathrm{La} /$ vacancy to perovskite A-cages so that configurations with different dimensionalities of the $\mathrm{Li}^{+}$diffusion pathway are included (see an example in Supplementary Fig. 4).

It is worth mentioning that the La order is accompanied by a change of symmetry in previous studies. For example, Li-poor compositions exhibit orthorhombic symmetry with high La-site occupancy in the La-rich layer, whereas the Li-rich composition has tetragonal symmetry, and the occupancy of La becomes homogeneous ${ }^{59-61}$. However, recent studies have shown that the symmetry with Li-rich composition is also orthorhombic with the $\mathrm{Cmmm}$ space group ${ }^{58,62,63}$. In order to understand the relationship between the La ordering and symmetry, configurations at $3 x=$ $0.125,0.2,0.25,0.3125$, and 0.35 are obtained in both hosts by group-subgroup transformation (Supplementary Table 7). Among all these structures, 100 symmetrically distinct ordered phases at each concentration with minimal electrostatic energies are selected for first-principles calculations. The ordered phases with the lowest formation energies are selected as the representative ordered ground states for further study.

As illustrated in Fig. 7a, it is found that the most stable configurations have an orthorhombic symmetry, confirming the recent experimental results ${ }^{63,64}$. Based on these ordered ground states (Supplementary Table 8), the occupation ratio of La is further explored. The average occupancy of La in the alternating La-poor and La-rich layers is illustrated in Fig. 7b. A clear ordering preference can be observed. This preference decreases with increasing $\mathrm{Li}$ content, which is consistent with the experimental results ${ }^{62}$.

Once the ordered ground states are determined, an analysis of the blocking effect of La on the migration pathway is performed. To further reveal the $\mathrm{Li}$ diffusion behavior in the $\mathrm{Li}_{3 x} \mathrm{La}_{2 / 3-x} \mathrm{TiO}_{3}$ systems, we calculate the energy barriers using the bond valence site energy (BVSE, see in Supplementary Methods) method as shown in Table $1^{65,66}$. At low Li concentrations of $3 x=0.125$ and 0.2 , the BVSE energy barriers of the ab-plane are much lower than that along the $c$ direction, which is consistent with the experimental result that $\mathrm{Li}$ ions diffuse in the ab-plane at low $\mathrm{Li}$ concentrations of $3 x<0.21^{67,68}$. This is caused by La ordering along the $c$-axis, as illustrated in Fig. $8 a, b$. One of the planes is fully occupied by La (La-rich plane), and the rest of La are located in the other plane (La-poor plane). Such alternating stacking between La-rich and La-poor planes also agrees well with the experiment results. In the high-Li-concentration region, i.e., at $3 x$ $=0.25,0.3125,0.35$, the La ions order arranges along the $a$-axis, as shown in Fig. 8c-e. In these systems, the BVSE energy barriers of the bc-plane are much lower than that along the direction, indicating that Li-ion diffusion in the bc-plane is unobstructed and that the pathway remains two-dimensional. This conclusion supports the experimental results that Li-ion diffusion is two dimensional below $127^{\circ} \mathrm{C}^{69}$. As discussed above, lithium-ion diffusion for all the $\mathrm{Li}_{3 x} \mathrm{La}_{2 / 3-x} \mathrm{TiO}_{3}$ systems is strongly anisotropic owing to the blocking effect of La. To eliminate the effect of diffusion anisotropy, it is useful to introduce another fast ion conductor (such as lithium silicate) into the grain boundary ${ }^{69}$.

\section{DISCUSSION}

In this work, we present that ordered ground states formed either during the preparation or the ion-intercalation process in several rechargeable battery materials, especially with transformed lattices or dilute alkali-ion concentrations, could be predicted using group-subgroup transformation. In $\mathrm{LiCoO}_{2}$, we solve the ordering problem for different sizes and shapes of supercells, including confirming the ordered ground states at $x=0.1667$, $0.3333,0.5$, and 0.6667 and resolving the previous conflict 

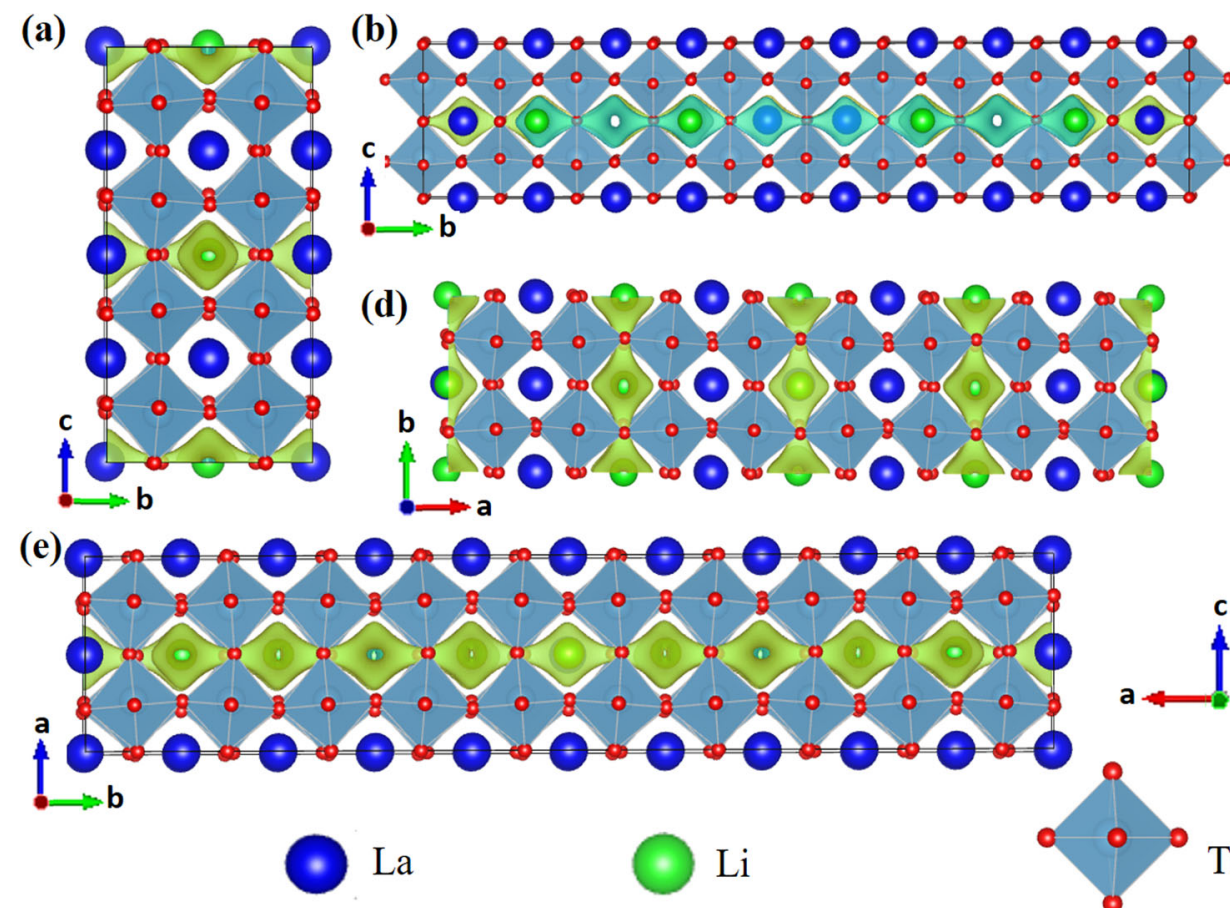

$\mathrm{Li}$
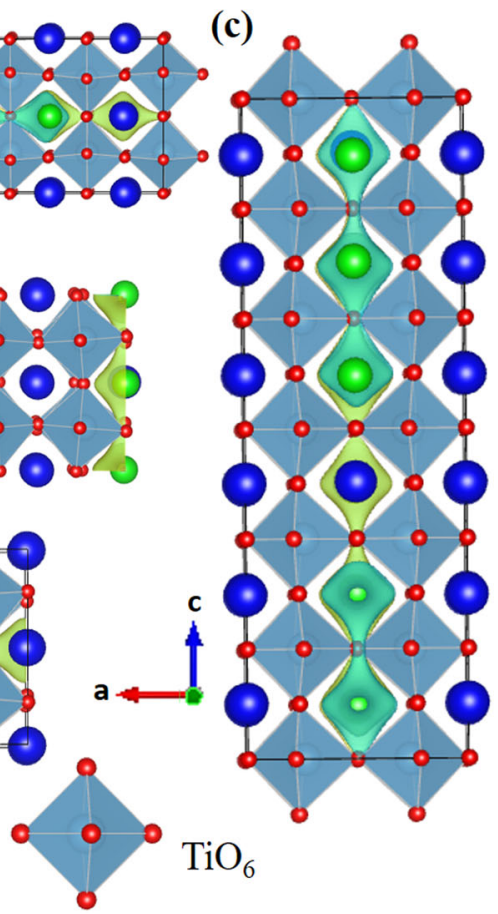

Fig. 8 Ordered ground states of $\mathrm{Li}_{3 x} \mathrm{La}_{2 / 3-x} \mathrm{TiO}_{3} .3 x=\mathbf{a} 0.125, \mathbf{b} \quad 0.2, \mathbf{c} 0.25, \mathbf{d ~} 0.3125$, and e 0.35 . Li-ion diffusion is anisotropic because of the blocking effect of La.
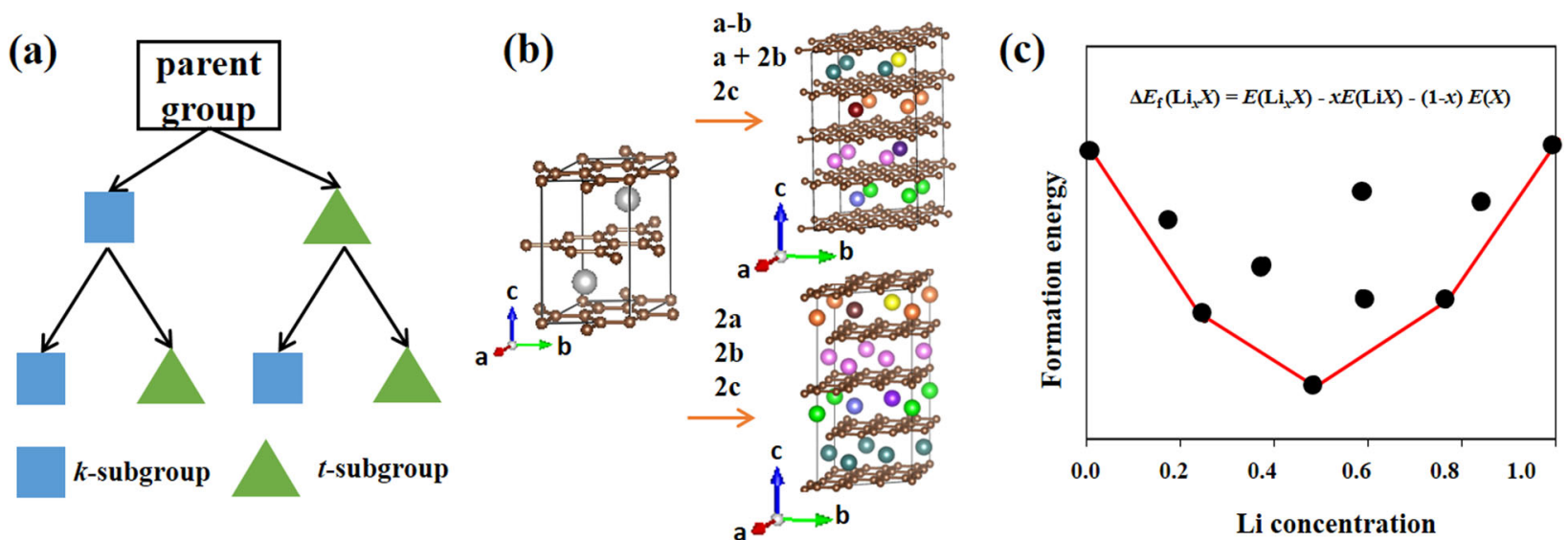

Fig. 9 Application of group-subgroup transformation for prediction of the ordered ground states. a Determination of subgroups and corresponding transformation matrix of the parent space group according to CELLSUB. b Subgroup structures are obtained by TRANSTRU with transformation matrix as an input. The splitting of Li position is illustrated by different colors. Vacancy and Li can occupy independent sites (illustrated by $\left.\mathrm{Li}_{x} \mathrm{C}_{6}\right)$. c Formation energies are obtained by first-principles calculations, and ordered ground states are determined by the convex hull.

concerning the ordered ground states of $\mathrm{Li}_{x} \mathrm{CoO}_{2}$ at $x=0.75$, $0.8333,0.8571$. And an entirely new $\mathrm{Li}_{0.875} \mathrm{CoO}_{2}$ ground state is identified at $x>0.75$. In $\mathrm{LiC}_{6}$, utilizing the Wyckoff splitting rule, we identify the new stable $\mathrm{Li}_{0.0625} \mathrm{C}_{6}$ with a stage IV structure as the most dilute phase, which has not been previously demonstrated in experimental and computational studies. Besides, this method also reveals the blocking effect of La on the diffusion anisotropy of Li. This method has also been successfully applied in the prediction of ordered phases in $\mathrm{K}_{x} \mathrm{Mn}_{7 / 9} \mathrm{Ti}_{2 / 9} \mathrm{O}_{2}{ }^{70}$. Moreover, partial replacement, such as substituting $\mathrm{Co}^{3+}$ with $\mathrm{Ni}^{2+}$ and $\mathrm{Mn}^{4+}$ in $\mathrm{Li}$ $\left(\mathrm{Ni}_{x} \mathrm{Mn}_{y} \mathrm{Co}_{1-x-y}\right) \mathrm{O}_{2}$ layered oxides (coined $\mathrm{NMC}$ ) would be an interesting topic for further investigation. It is worth mentioning that in the cases where framework ions can leave their original site and migrate to the site of mobile ions, such as the transition of $\mathrm{LiCoO}_{2}$ into cubic $\mathrm{Li}_{0.5} \mathrm{CoO}_{2}$, the phase transition is more complicated and thus not discussed here. In addition, constraints on crystal systems and $k$-indexes have to be implemented in practical applications, ensuring that the group-subgroup transformation method is especially efficient when it comes to solving ordered arrangements.

In addition to atomic ordering, this method is also valuable for other ordering problems such as systems with magnetism, which is closely related to the ordering of charges. It is also potentially applicable to materials such as light-emitting $\mathrm{Cs}_{2} \ln ^{\prime} \operatorname{In}^{\prime \prime \prime} \mathrm{Cl}_{6}$ with the charge ordering of $\mathrm{In}^{\prime} / / \mathrm{In}^{\prime \prime \prime}$ and pristine $\mathrm{Cr}_{2} \mathrm{Ge}_{2} \mathrm{Te}_{6}$ (twodimensional van der Waals material) with ferromagnetic ordering $^{71,72}$. Identifying the charge ordering, ferroelectricity, or magnetic ordering and their evolutions would be comparatively difficult but desired. Thus, in addition to rechargeable batteries, we believe the group-subgroup transformation can also be 
applied to other areas such as ferroelectrics and other chargeordering-related materials.

\section{METHODS}

\section{Determining subgroups}

Group-subgroup transformation starts from a space group of a highly symmetrical parent structure $(G)$. The extraction of alkali-ions from the host structure results in the breaking of symmetry from either the point group $(P(G))$ or the translation group $(T(G))$ to maximal $t$-subgroups or $k$-subgroups, respectively $(H)$. Maximal $k$-subgroups can be further classified into Loss of centering translations, Non-isomorphism, and Isomorphism. An example of these maximal subgroups is shown in Supplementary Methods. Then, each of the subgroups further acts as a new highly symmetrical parent group and has its maximal $t$-subgroups or $k$-subgroups. Therefore, the group-subgroup relations can eventually be divided into a number of steps $G>H_{1}>H_{2}>\ldots>H$, with each involving either a maximal $t$-subgroup or $k$-subgroup, as illustrated in Fig. 9a. Thus, the parent structure can be consecutively degenerated to lower symmetry subgroups. The reduction factor in the point group is characterized by the $t$-index (Eq. 1), which should be a divisor of the order of $P(G)(|P(G)|)$. The reduction factor in the translation group is further characterized by the $k$-index (Eq. 2), which should be a divisor of the order of $T(G)(|T(G)|$

$t-$ index $=|P(G)| /|P(H)|$

$k-$ index $=|T(G)| /|T(H)|$

For each step, the transformation matrix that determines new lattices of the subgroup is compiled in the International Table of Crystallography, and can also be accessed by the Bilbao Crystallographic Server ${ }^{73,74}$. Finally, $H$ transforms into the triclinic group $P 1$, corresponding to the removal of all symmetry and enumeration of all possible configurations. Given that such a low symmetry causes a large number of configurations, it is necessary to constrain the search to certain crystal systems that are detected by experiments and an upper limit of $k$-index ( $k$-index also indicates the multiplication factor relating the volume of the primitive structure of the subgroup to that of the original prototype structure) ${ }^{75}$.

In addition, the transformation of the lattice may occur because symmetry operations in the subgroup inevitably change. In each of the transformation matrix-column pairs $(\boldsymbol{P}, \boldsymbol{p})$, the $3 \times 3$ square matrix $\boldsymbol{P}$ transforms the conventional basis $(\mathbf{a}, \mathbf{b}, \mathbf{c})$ denoted as $G$ into another conventional basis $H$ (Eq. 3$)^{74}$. The column $\boldsymbol{p}$ of coordinates of the origin $\mathrm{O}$ of $H$ is referred to the coordinate system of $G$ and is called the origin shift.

$$
\begin{aligned}
& \mathbf{a}^{\prime}=P_{11} \mathbf{a}+P_{21} \mathbf{b}+P_{31} \mathbf{c} \\
& \mathbf{b}^{\prime}=P_{12} \mathbf{a}+P_{22} \mathbf{b}+P_{32} \mathbf{c} \\
& \mathbf{c}^{\prime}=P_{13} \mathbf{a}+P_{23} \mathbf{b}+P_{33} \mathbf{c}
\end{aligned}
$$

\section{Splitting of Wyckoff position for each pair $\boldsymbol{G}>\boldsymbol{H}$}

In the parent structure with high symmetry, atoms are symmetrically equivalent if they share the same Wyckoff position under the manipulation of symmetry. However, in the subgroup, because of the reduction of symmetry, these atoms may become non-equivalent. This allows the high-symmetry Wyckoff position of alkali-ion which corresponds to the high-symmetry group of the prototype to split into different sets of positions in the subgroup (Fig. $9 \mathrm{~b}$ and Supplementary Fig. 6e). Each subset can then be occupied independently by an alkali-ion or a vacancy, resulting in subgroup configurations with different alkali-ion contents. The theoretical relation of the Wyckoff positions for a group-subgroup pair $G>H$ has been demonstrated by Wondratschek et al. ${ }^{74}$.

\section{Ground states determination}

After configurations are obtained by assigning alkali-ion/vacancy to the independent sets of positions, the StructureMatcher utility in Pymatgen is employed to exclude identical arrangements ${ }^{76}$. It compares two structures by reducing them to primitive cells and evaluates whether the maximum root means square displacement is less than a predefined tolerance cutoff. This method can effectively distinguish the nonidentical structures and has proven useful in many previous works ${ }^{77,78}$. Firstprinciples calculations are then performed to obtain the formation energies and determine the ordered ground states (for calculation details, see Supplementary Methods) $)^{29,79}$. Taking the $\mathrm{Li}_{x} \mathrm{CoO}_{2}$ compound as an example, the formation energy of a given $\mathrm{Li} /$ vacancy configuration with content $x$ in $\mathrm{Li}_{x} \mathrm{CoO}_{2}$ is defined as

$\Delta E_{\mathrm{f}}\left(\mathrm{Li}_{x} \mathrm{CoO}_{2}\right)=E\left(\mathrm{Li}_{x} \mathrm{CoO}_{2}\right)-x E\left(\mathrm{LiCoO}_{2}\right)-(1-x) E\left(\mathrm{CoO}_{2}\right)$

where $E\left(\mathrm{Li}_{x} \mathrm{CoO}_{2}\right)$ is the total energy of the configuration per $\mathrm{Li}_{x} \mathrm{CoO}_{2}$ formula unit, and $E\left(\mathrm{LiCoO}_{2}\right)$ and $E\left(\mathrm{CoO}_{2}\right)$ are the energies of $\mathrm{LiCoO}_{2}$ and $\mathrm{CoO}_{2}$ in the $\mathrm{O} 3$ host, respectively. The formation energy reflects the relative stability of that structure concerning phase separation into a fraction $x$ of $\mathrm{LiCoO}_{2}$ and a fraction $(1-x)$ of $\mathrm{CoO}_{2}$.

Procedures of group-subgroup transformation and bond valence site energy calculations are implemented in the high-throughput computational platform for battery materials ${ }^{80}$.

\section{DATA AVAILABILITY}

The authors declare that the main data (3779 and 5811 ordered phases, obtained by group-subgroup transformation, during ion-intercalation/extraction processes of $\mathrm{Li}_{x} \mathrm{CoO}_{2}(0<x<1)$ and $\mathrm{Li}_{x} \mathrm{C}_{6}(0<x<1)$, respectively, and the nonidentical sorts of these ordered phases) supporting the findings of this study are available within the article and Supplementary Tables 12 and 13. All source files (CIF and POSCAR) of all ordered phases are uploaded to the repository: https://github.com/ shuhebing/gsop.

\section{CODE AVAILABILITY}

All source codes of the method that are implemented in Python are uploaded to the repository: https://github.com/shuhebing/gsop.

Received: 22 March 2021; Accepted: 21 October 2021;

Published online: 12 November 2021

\section{REFERENCES}

1. Nguyen, V. H. \& Kim, Y. H. Recent advances in cathode and anode materials for lithium ion batteries. Appl. Chem. Eng. 29, 635-644 (2018).

2. Kim, T., Song, W. D., Son, Y., Ono, L. K. \& Qi, Y. Lithium-ion batteries: outlook on present, future, and hybridized technologies. J. Mater. Chem. A 7, 2942-2964 (2019).

3. Islam, M. S. \& Fisher, C. A. Lithium and sodium battery cathode materials: computational insights into voltage, diffusion, and nanostructural properties. Chem. Soc. Rev. 43, 185-204 (2014).

4. Zu, C. X. \& Li, H. Thermodynamic analysis on energy densities of batteries. Energ. Environ. Sci. 4, 2614-2624 (2011).

5. Kganyago, K. Ab initio calculation of the voltage profile for $\mathrm{LiC}_{6}$. Solid State lon. 159, 21-23 (2003).

6. He, P., Yu, H., Li, D. \& Zhou, H. Layered lithium transition metal oxide cathodes toward high energy lithium-ion batteries. J. Mater. Chem. 22, 3680-3695 (2012).

7. Rozier, P. \& Tarascon, J. M. Li-rich layered oxide cathodes for next-generation Li-ion batteries: chances and challenges. J. Electrochem. Soc. 162, A2490-A2499 (2015).

8. Liu, Y. et al. Monoclinic phase Na3Fe2(PO4)3: synthesis, structure, and electrochemical performance as cathode material in sodium-ion batteries. ACS Sustain. Chem. Eng. 5, 1306-1314 (2016).

9. Deng, Y. et al. Crystal structures, local atomic environments, and ion diffusion mechanisms of scandium-substituted sodium superionic conductor (NASICON) solid electrolytes. Chem. Mater. 30, 2618-2630 (2018).

10. Roy, S. \& Kumar, P. P. Influence of cationic ordering on ion transport in NASICONs: molecular dynamics study. Solid State Ion. 253, 217-222 (2013).

11. Roy, S. \& Kumar, P. P. Influence of Si/P ordering on $\mathrm{Na}^{+}$transport in NASICONs. Phys. Chem. Chem. Phys. 15, 4965-4969 (2013).

12. Kabbor, H., Coillot, D., Colmont, M., Masquelier, C. \& Mentre, O. a-Na $\mathrm{Na}_{2}\left(\mathrm{PO}_{4}\right)_{3}$ $(M=\mathrm{Ti}, \mathrm{Fe})$ : absolute cationic ordering in NASICON-type phases. J. Am. Chem. Soc. 133, 11900-11903 (2011).

13. Kozinsky, B. et al. Effects of sublattice symmetry and frustration on ionic transport in garnet solid electrolytes. Phys. Rev. Lett. 116, 055901 (2016).

14. Lu, Z. et al. Modulating nanoinhomogeneity at electrode-solid electrolyte interfaces for dendrite-proof solid-state batteries and long-life memristors. Adv. Energy Mater. 11, 2003811 (2021).

15. Xu, M. et al. Mechanisms of $\mathrm{Li}^{+}$transport in garnet-type cubic $\mathrm{Li}_{3+x} \mathrm{La}_{3} \mathrm{M}_{2} \mathrm{O}_{12}(M=\mathrm{Te}$ Nb, Zr). Phys. Rev. B. 85, 052301 (2012).

16. $\mathrm{Kim}, \mathrm{H}$. et al. Investigation of potassium storage in layered P3-type $\mathrm{K} 0.5 \mathrm{MnO} 2$ cathode. Adv. Energy Mater. 29, 1702480 (2017). 
17. Hwang, J. Y., Kim, J., Yu, T. Y. \& Sun, Y. K. A new P2-type layered oxide cathode with extremely high energy density for sodium-ion batteries. Adv. Energy Mater. 9, 1803346 (2019)

18. Vaalma, C., Giffin, G. A., Buchholz, D. \& Passerini, S. Non-aqueous K-ion battery based on layered $\mathrm{K} 0.3 \mathrm{MnO} 2$ and hard carbon/carbon black. J. Electrochem. Soc. 163, A1295-A1299 (2016).

19. Chen, T., Gautam, G. S., Huang, W. \& Ceder, G. First-principles study of the voltage profile and mobility of $\mathrm{Mg}$ intercalation in a chromium oxide spinel. Chem. Mater. 30, 153-162 (2018).

20. Meng, Y. S., Hinuma, Y. \& Ceder, G. An investigation of the sodium patterning in $\mathrm{Na}_{x} \mathrm{CoO}_{2}(0.5 \leq x \leq 1)$ by density functional theory methods. J. Chem. Phys. 128, 104708 (2008).

21. Berthelot, R., Carlier, D. \& Delmas, C. Electrochemical investigation of the P2$\mathrm{Na}_{x} \mathrm{CoO}_{2}$ phase diagram. Nat. Mater. 10, 74-80 (2011)

22. $\mathrm{Xu}$, J. et al. Recent progress in graphite intercalation compounds for rechargeable metal (Li, Na, K, Al)-ion batteries. Adv. Sci. 4, 1700146 (2017).

23. Sun, Y. et al. Recent progress in lithium lanthanum titanate electrolyte towards all solid-state lithium ion secondary battery. Crit. Rev. Solid State 44, 265-282 (2019).

24. Matsunaga, T. et al. Comprehensive elucidation of crystal structures of lithiumintercalated graphite. Carbon 142, 513-517 (2019).

25. Derosa, P. A. \& Balbuena, P. B. A lattice-gas model study of lithium intercalation in graphite. J. Electrochem. Soc. 146, 3630-3638 (1999).

26. Kalikmanov, V. I., Koudriachova, M. V. \& de Leeuw, S. W. Lattice-gas model for intercalation compounds. Solid State Ion. 136, 1373-1378 (2000).

27. Lu, Z. \& Ciucci, F. Anti-perovskite cathodes for lithium batteries. J. Mater. Chem. A 6, 5185-5192 (2018)

28. Hart, G. L. W. \& Forcade, R. W. Generating derivative structures from multilattices: algorithm and application to hcp alloys. Phys. Rev. B 80, 014120 (2009).

29. Kim, S. et al. First-Principles study of lithium cobalt spinel oxides: Correlating structure and electrochemistry. ACS Appl. Mater. Inter. 10, 13479-13490 (2018).

30. Wu, Q., He, B., Song, T., Gao, J. \& Shi, S. Cluster expansion method and its application in computational materials science. Comp. Mater. Sci. 125, 243-254 (2016).

31. Lu, Z., Zhu, B., Shires, B. W. B., Scanlon, D. O. \& Pickard, C. J. Ab initio random structure searching for battery cathode materials. J. Chem. Phys. 154, 174111 (2021).

32. Sendek, D. et al. Holistic computational structure screening of more than 12000 candidates for solid lithium-ion conductor materials. Energ. Environ. Sci. 10, 306-320 (2017)

33. Hafner, J. Ab-initio simulations of materials using VASP: density-functional theory and beyond. J. Comput. Chem. 29, 2044-2078 (2008).

34. Yahia, H. B., Shikano, M. \& Kobayashi, H. Phase transition mechanisms in $\mathrm{Li}_{x} \mathrm{CoO}_{2}$ $(0.25 \leq x \leq 1)$ based on group-subgroup transformations. Chem. Mater. 25, 3687-3701 (2013)

35. Shao-Horn, Y., Levasseur, S., Weill, F. \& Delmas, C. Probing lithium and vacancy ordering in $\mathrm{O} 3$ layered $\mathrm{Li}_{x} \mathrm{CoO}_{2}(x \approx 0.5)$ : an electron diffraction study. J. Electrochem. Soc. 150, A366 (2003).

36. Blangero, M., Decourt, R. \& Carlier, D. First experimental evidence of potassium ordering in layered $\mathrm{K}_{4} \mathrm{Co}_{7} \mathrm{O}_{14}$. Inorg. Chem. 44, 9299-9304 (2005).

37. Van der Ven, A., Aydinol, M. K., Ceder, G., Kresse, G. \& Hafner, J. First-principles investigation of phase stability in $\mathrm{Li}_{x} \mathrm{CoO}_{2}$. Phys. Rev. B 58, 2975 (1998).

38. Wolverton, C. \& Zunger, A. First-principles prediction of vacancy order-disorder and intercalation battery voltages in $\mathrm{Li}_{x} \mathrm{CoO}_{2}$. Phys. Rev. Lett. 81, 606 (1998).

39. Takahashi, Y. et al. Structure and electron density analysis of electrochemically and chemically delithiated $\mathrm{LiCOO}_{2}$ single crystals. J. Solid State Chem. 180, 313-321 (2007).

40. Amatucci, G. G., Tarascon, J. M. \& Klein, L. C. $\mathrm{CoO}_{2}$, the end member of the $\mathrm{Li}_{x} \mathrm{CoO}_{2}$ solid solution. J. Electrochem. Soc. 143, 1114 (1996).

41. Van der Ven, A., Aydinol, M. K. \& Ceder, G. First-Principles evidence for stage ordering in $\mathrm{Li}_{x} \mathrm{CoO}_{2}$. J. Electrochem. Soc. 145, 2149 (1998).

42. Chen, Z., Lu, Z. \& Dahn, J. R. Staging phase transitions in $\mathrm{Li}_{x} \mathrm{CoO}_{2}$. J. Electrochem. Soc. 149, A1604 (2002).

43. Reimers, J. N. \& Dahn, J. R. Electrochemical and in situ X-ray diffraction studies of lithium intercalation in $\mathrm{Li}_{x} \mathrm{CoO}_{2}$. J. Electrochem. Soc. 139, 2091 (1992).

44. Luo, W. et al. Potassium ion batteries with graphitic materials. Nano Lett. 15, 7671-7677 (2015)

45. Sun, Y., Lu, X., Xiao, R., Li, H. \& Huang, X. Kinetically controlled lithium-staging in delithiated $\mathrm{LiFePO}_{4}$ driven by the Fe center mediated interlayer $\mathrm{Li}-\mathrm{Li}$ interactions. Chem. Mater. 24, 4693-4703 (2012).

46. Persson, K., Hinuma, Y., Meng, Y. S., Van der Ven, A. \& Ceder, G. Thermodynamic and kinetic properties of the Li-graphite system from first-principles calculations. Phys. Rev. B 82, 125416 (2010).

47. Dahn, J. R. Phase diagram of $\mathrm{Li}_{x} \mathrm{C}_{6}$. Phys. Rev. B. 44, 9170 (1991).

48. Heß, M. \& Novák, P. Shrinking annuli mechanism and stage-dependent rate capability of thin-layer graphite electrodes for lithium-ion batteries. Electrochim. Acta 106, 149-158 (2013).
49. Lenchuk, O., Adelhelm, P. \& Mollenhauer, D. New insights into the origin of unstable sodium graphite intercalation compounds. Phys. Chem. Chem. Phys. 21, 19378-19390 (2019)

50. Wang, Z., Selbach, S. M. \& Grande, T. Van der Waals density functional study of the energetics of alkali metal intercalation in graphite. RSC Adv. 4, 4069-4079 (2014).

51. Oka, H. et al. Changes in the stage structure of Li-intercalated graphite electrode at elevated temperatures. J. Power Sources 482, 228926 (2021).

52. Allart, D., Montaru, M. \& Gualous, H. Model of lithium intercalation into graphite by potentiometric analysis with equilibrium and entropy change curves of graphite electrode. J. Electrochem. Soc. 165, A380-A387 (2018).

53. Kostecki, R. \& McLarnon, F. Microprobe study of the effect of Li intercalation on the structure of graphite. J. Power Sources 119-121, 550-554 (2003).

54. Yazami, R. \& Touzain, P. A reversible graphite-lithium negative electrode for electrochemical generators. J. Power Sources 9, 365-371 (1983).

55. Mitsuishi, K. et al. Nazca lines by La ordering in $\mathrm{La}_{2 / 3-x} \mathrm{Li}_{3 x} \mathrm{TiO}_{3}$ ion-conductive perovskite. Appl. Phys. Lett. 101, 073903 (2012).

56. Catti, M. Short-range order and $\mathrm{Li}^{+}$ion diffusion mechanisms in $\mathrm{Li}_{5} \mathrm{La}_{9}\left(\mathrm{TiO}_{3}\right)_{16}$ (LLTO). Solid State lon. 183, 1-6 (2011).

57. Gao, X. et al. Cation ordering in A-site-deficient Li-ion conducting perovskites $\mathrm{La}_{(1-x) / 3} \mathrm{Li}_{x} \mathrm{NbO}_{3}$. J. Mater. Chem. A 3, 3351-3359 (2015).

58. Cheng, Y. Q. et al. An integrated approach for structural characterization of complex solid state electrolytes: the case of lithium lanthanum titanate. J. Mater. Chem. A 2, 2418-2426 (2014).

59. Ibarra, J. Influence of composition on the structure and conductivity of the fast ionic conductors $\mathrm{La}_{2 / 3-x} \mathrm{Li}_{3 x} \mathrm{TiO}_{3}(0.03 \leq x \leq 0.167)$. Solid State lon. 134, 219-228 (2000).

60. Fourquet, J. L., Duroy, H. \& Crosnier-Lopez, M. P. Structural and microstructural studies of the series $\mathrm{La}_{2 / 3-x} \mathrm{Li}_{3 x} \mathrm{TiO}_{3}$. J. Solid State Chem. 127, 283-294 (1996).

61. Wu, J. et al. A review on structural characteristics, lithium ion diffusion behavior and temperature dependence of conductivity in perovskite-type solid electrolyte $\mathrm{Li}_{3 x} \mathrm{La}_{2 / 3-x} \mathrm{TiO}_{3}$. Funct. Mater. Lett. 10, 1730002 (2017).

62. Okumura, T. et al. Effect of average and local structures on lithium ion conductivity in $\mathrm{La}_{2 / 3-x} \mathrm{Li}_{3 x} \mathrm{TiO}_{3}$. J. Mater. Chem. 21, 10195 (2011).

63. Inaguma, Y., Katsumata, T., Itoh, M., Morii, Y. \& Tsurui, T. Structural investigations of migration pathways in lithium ion-conducting $\mathrm{La}_{2 / 3-x} \mathrm{Li}_{3 x} \mathrm{TiO}_{3}$ perovskites. Solid State lon. 177, 3037-3044 (2006).

64. Garciamartin, S., Alariofranco, M. A., Ehrenberg, H., Rodriguezcarvajal, J. \& Amador, U. Crystal structure and microstructure of some $\mathrm{La}_{2 / 3-x} \mathrm{Li}_{3 x} \mathrm{TiO}_{3}$ oxides: an example of the complementary use of electron diffraction and microscopy and synchrotron X-ray diffraction to study complex materials. J. Am. Chem. Soc. 126, 3587-3596 (2004)

65 . He, B. et al. A highly efficient and informative method to identify ion transport networks in fast ion conductors. Acta Mater. 203, 116490 (2021).

66 . He, B. et al. CAVD, toward better characterization of void space for ionic transport analysis. Sci. Data 7, 153 (2020).

67. Maruyama, Y., Ogawa, H., Kamimura, M., Ono, S. \& Kobayashi, M. Dynamical properties and electronic structure of $(\mathrm{LaLi}) \mathrm{TiO}_{3}$ conductors. lonics 14, 357-361 (2008).

68. Inaguma, Y. et al. High ionic conductivity in lithium lanthanum titanate. Solid State Commun. 86, 689-693 (1993).

69. Chen, C. \& Du, J. Lithium ion diffusion mechanism in lithium lanthanum titanate solid-state electrolytes from atomistic simulations. J. Am. Ceram. Soc. 98, 534-542 (2015).

70. $\mathrm{Xu}, \mathrm{Y}$. S. et al. Enabling reversible phase transition on $\mathrm{K}_{5 / 9} \mathrm{Mn}_{7 / 9} \mathrm{Ti}_{2 / 9} \mathrm{O}_{2}$ for highperformance potassium-ion batteries cathodes. Energy Storage Mater. 31, 20-26 (2020).

71. Gong, C. et al. Discovery of intrinsic ferromagnetism in two-dimensional van der Waals crystals. Nature 546, 265-269 (2017).

72. Seidel, S. \& Pöttgen, R. Group-subgroup schemes for $\mathrm{MoNi}_{4}, \mathrm{Nb}_{4} \mathrm{~N}_{5}, \mathrm{~K}_{x} \mathrm{Fe}_{2-y} \mathrm{Se}_{2}$, $\mathrm{Nd}_{10} \mathrm{Au}_{3} \mathrm{As}_{8} \mathrm{O}_{10}$, and $\mathrm{Cs}_{\mathrm{InCl}}$ : i5 superstructures of $14 / \mathrm{m}$ allowing atom, charge or vacancy ordering. Z. Krist.-Cryst. Mater. 235, 29-39 (2020).

73. Aroyo, M. I. et al. Bilbao crystallographic server: I. Databases and crystallographic computing programs. Z. Krist.-Cryst. Mater. 221, 15-27 (2006).

74. Hahn, T., Shmueli, U., Arthur, J. W. (eds) International Tables for Crystallography, Vol. 1 (Reidel, 1983).

75. Kozinsky B. Transport in frustrated and disordered solid electrolytes. In: Andreoni W., Yip S. (eds) Handbook of Materials Modeling: Applications: Current and Emerging Materials, Springer International Publishing, Cham, pp. 1-20 (2018).

76. Ong, S. P. et al. Python Materials Genomics (pymatgen): a robust, open-source python library for materials analysis. Comp. Mater. Sci. 68, 314-319 (2013).

77. Li, A., Bueno-Perez, R., Wiggin, S. \& Fairen-Jimenez, D. Enabling efficient exploration of metal-organic frameworks in the Cambridge Structural Database. CrystEngComm 22, 7152-7161 (2020). 
78. G. Cheon, L. Yang, K. McCloskey, E. J. Reed, E. D. Cubuk. Crystal structure search with random relaxations using graph networks. Preprint at https://arxiv.org/abs/ 2012.02920 (2020)

79. Wang, L., Maxisch, T. \& Ceder, G. Oxidation energies of transition metal oxides within the GGA+U framework. Phys. Rev. B 73, 195107 (2006).

80. He, B. et al. High-throughput screening platform for solid electrolytes combining hierarchical ion-transport prediction algorithms. Sci. Data 7, 151 (2020).

\section{ACKNOWLEDGEMENTS}

This work is supported by the National Natural Science Foundation of China (Nos. 11874254, 51622207) and the National Key Research and Development Program of China (No. 2017YFB0701600). All the computations are performed on the highperformance computing platform provided by the High-Performance Computing Center of Shanghai University.

\section{AUTHOR CONTRIBUTIONS}

Y.R. prepares the manuscript and analyzes the data. D.W., Z.Z., B.P., and Z.L. help to perform the analysis with constructive discussions. B.L., Y.R., and W. S perform the first-principles calculations and check the data. P.M. and B.H. develop the BVSE tool. Y.L., Z.L., X.L., and B.H.L. help to revise the manuscript. S.S. is the leader in this manuscript and contributes to the conception of the study. All authors participate in discussing the results and comments on the manuscript.

\section{COMPETING INTERESTS}

The authors declare no competing interests.

\section{ADDITIONAL INFORMATION}

Supplementary information The online version contains supplementary material available at https://doi.org/10.1038/s41524-021-00653-y.

Correspondence and requests for materials should be addressed to Siqi Shi.

Reprints and permission information is available at http://www.nature.com/ reprints

Publisher's note Springer Nature remains neutral with regard to jurisdictional claims in published maps and institutional affiliations.

Open Access This article is licensed under a Creative Commons Attribution 4.0 International License, which permits use, sharing, adaptation, distribution and reproduction in any medium or format, as long as you give appropriate credit to the original author(s) and the source, provide a link to the Creative Commons license, and indicate if changes were made. The images or other third party material in this article are included in the article's Creative Commons license, unless indicated otherwise in a credit line to the material. If material is not included in the article's Creative Commons license and your intended use is not permitted by statutory regulation or exceeds the permitted use, you will need to obtain permission directly from the copyright holder. To view a copy of this license, visit http://creativecommons. org/licenses/by/4.0/.

(c) The Author(s) 2021, corrected publication 2022 